\title{
Assessing Endocardial Activation from Bipolar Intra-Ventricular Electrodes: A Simulation Study
}

\author{
Peter R Johnston ${ }^{1}$, Gerald Fischer ${ }^{2}$ \\ ${ }^{1}$ Griffith University, Brisbane, Australia \\ ${ }^{2}$ AFreeze GmbH, Innsbruck, Austria
}

\begin{abstract}
Assessment of endocardial activation using filtered catheter-recorded electrograms has become a standard clinical technique. This study aims to assess factors affecting the accuracy of this process.

The simulation study confirmed the ability of catheterbased signals to assess relative ("early vs. late") timing of ventricular activation. However, absolute errors were high, amounting to $10 \%$ of the activation interval. Computer-based investigations provide insight into how relevant parameters (catheter size and angle, filtering) affect accuracy. This may allow for further improvement of catheter-based techniques.
\end{abstract}

\section{Introduction}

Catheter-based electro-anatomical mapping of cardiac activation has been a clinical standard for almost two decades. In order to exclude far field potentials and signal noise from the analysis, band pass filtered bipolar electrograms are frequently used. However, there are no theoretical underpinnings for methods that define reliable markers for the underlying cellular activation in the bipolar signals [1]. In practice, signals are annotated by trained experts [2], but various strategies for signal annotation are in use.

The aim of this study is to assess the accuracy of popular annotation strategies used in catheter-based activation mapping techniques. Computer modelling is used to compute membrane potentials (activation on cellular level) and extracellular potentials (catheter signals) for accurate comparison. The influence of electrode dimensions and filtering of the bipolar signals is included in the model to closely approximate the clinical situation.

\section{Methods}

The simulation model for this study is a simple halfellipsoid model of a left ventricle filled with blood. The simplified model is represented by the parametric equa- tions [3]:

$$
\begin{array}{cc}
x=a(r) \cos \theta \cos \phi & -\pi \leq \phi \leq \pi \\
y=b(r) \cos \theta \sin \phi & 0 \leq \theta \leq \frac{\pi}{2} \\
z=c(r) \sin \theta & 0 \leq r \leq 1
\end{array}
$$

where $a(r)=a_{1}+r\left(a_{2}-a_{1}\right), b(r)=b_{1}+r\left(b_{2}-b_{1}\right)$ and $c(r)=c_{1}+r\left(c_{2}-c_{1}\right)$. For this study, $a_{1}=b_{1}=2$, $a_{2}=b_{2}=3, c_{1}=5$ and $c_{2}=6$ (all dimensions are in centimetres). In the walls of the ventricle, the cardiac fibres rotate linearly with depth through an angle of $120^{\circ}$ anticlockwise from the epicardium to the endocardium, with the endocardial fibre offset $-45^{\circ}$ from the positive $z$-axis.

Electric potentials were recorded using a representation of a 7Fr mapping and ablation catheter, placed at varying locations around the ventricle, with the tip in contact with the endocardium. The probe consisted of four recording electrodes with insulating rings in between. It had a hemispherically-shaped tip and all dimensions are shown in Figure 1. The probe was placed in contact with the endocardium, 0.6 of the distance from the base to the apex of the ventricle and oriented such that it made a $15^{\circ}$ angle with the positive $z$-axis (see Figure 2).

To commence propagation, a $1 \mathrm{~mm}^{3}$ region of tissue adjacent to the endocardium and placed 0.3 of the distance from the base to the apex in the $x-z$ plane of the ventricle, was paced for $2 \mathrm{~ms}$ with a $0.25 \mathrm{~mA} / \mathrm{mm}^{3}$ current. Dif-

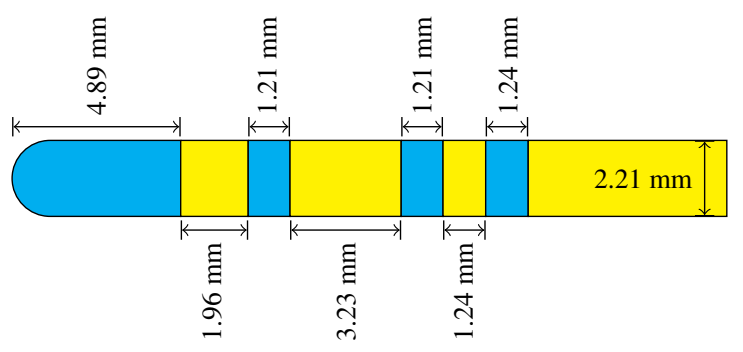

Figure 1. Schematic of probe used in the simulations, including the dimensions. The cyan regions indicate electrodes and the yellow regions are the insulated part of the probe. 


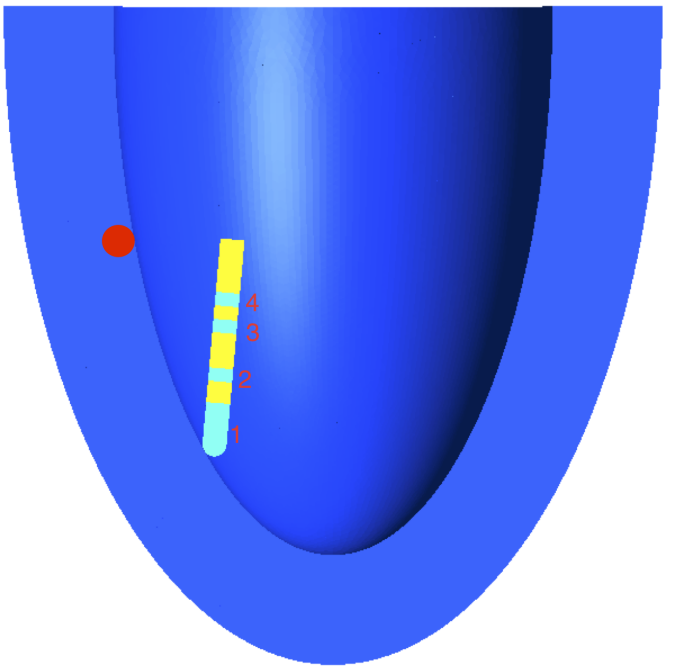

$\underline{X}_{Z}^{Y}$

Figure 2. Model configuration showing the position and orientation of the probe. The numbers indicate the electrodes referred to in the text and the red dot indicates the approximate location of the stimulus.

ferent simulations were performed as the probe was moved around the endocardium in 30 degree increments to simulate taking measurements in different places. Potentials were recorded at the four electrodes in the probe but, from these, only one bipolar electrogram was used in this study, by taking the difference in potentials on electrodes 1 and 2 .

To model propagation in the cardiac tissue, the bidomain model [4] was used. In this model, electric potentials in the intracellular and extracellular spaces, $\phi_{i}$ and $\phi_{e}$, respectively, are governed by the equations

$$
\nabla \cdot\left(\mathbf{M}_{i}+\mathbf{M}_{e}\right) \nabla \phi_{e}=-\nabla \cdot \mathbf{M}_{i} \nabla \phi_{m}
$$

and

$\nabla \cdot \mathbf{M}_{i} \nabla \phi_{e}+\nabla \cdot \mathbf{M}_{i} \nabla \phi_{m}=\beta\left(C_{m} \frac{\partial \phi_{m}}{\partial t}+I_{i o n}\left(\phi_{m}\right)\right)-f_{i s}$

where $\phi_{m}$ is the transmembrane potential, $\phi_{m}=\phi_{i}-\phi_{e}, \beta$ is the surface area to volume ratio for the cell $\left(2000 \mathrm{~cm}^{-1}\right)$, $C_{m}$ is the membrane capacitance $\left(1 \mu \mathrm{F} / \mathrm{cm}^{2}\right)$ and $f_{i s}$ is the applied stimulus in the intracellular space. The function $I_{\text {ion }}\left(\phi_{m}\right)$ represents the transmembrane current, which depends on the transmembrane potential distribution and, in this case, is governed by the model of ten Tusscher et al. [5]. The matrices $\mathbf{M}_{i}$ and $\mathbf{M}_{e}$ are conductivity tensors, which, in general, can be written as

$$
\mathbf{M}_{h}=\mathbf{A G}_{h} \mathbf{A}^{T}
$$

for $h=i$ (intracellular) and $h=e$ (extracellular). The matrix $\mathbf{A}$ represents the local direction of the fibres and $\mathbf{G}_{h}$ is a diagonal matrix containing the longitudinal $\left(g_{h l}\right)$, transverse $\left(g_{h t}\right)$ and normal $\left(g_{h n}\right)$ tissue conductivities along the diagonal. For these simulations, the following conductivity values were used [6]: $g_{i l}=2.4, g_{e l}=2.4$, $g_{i t}=0.35, g_{e t}=2.0, g_{i n}=0.08$ and $g_{e n}=1.1$ (all with units of $\mathrm{mS} / \mathrm{cm}$ ). The blood conductivity, $g_{b}$, was set at 6.7 $\mathrm{mS} / \mathrm{cm}$ [7], with the conductivity of the electrodes, $g_{\ell}$, set at $1000 g_{b}$ and that of the insulating parts of the probe at $0.0001 g_{b}$.

In the blood cavity, which includes the probe, the potential was governed by Laplace's equation:

$$
\nabla^{2} \phi_{b}=0 \text { and } \nabla^{2} \phi_{\ell}=0
$$

where $\phi_{b}$ represents the potential in the blood and $\phi_{\ell}$ represents the potential on the electrodes.

To complete the model, a set of boundary and initial conditions was required. It was assumed that the outer surface of the model was insulated, that is

$$
\frac{\partial \phi_{e}}{\partial n}=\frac{\partial \phi_{i}}{\partial n}=\frac{\partial \phi_{b}}{\partial n}=0 .
$$

There was also continuity of potential and current between the blood and the tissue,

$$
\phi_{e}=\phi_{b}, \quad g_{e n} \frac{\partial \phi_{e}}{\partial n}=g_{b} \frac{\partial \phi_{b}}{\partial n}
$$

as well as between the blood and the electrodes

$$
\phi_{\ell}=\phi_{b}, \quad g_{\ell} \frac{\partial \phi_{\ell}}{\partial n}=g_{b} \frac{\partial \phi_{b}}{\partial n} .
$$

Finally, it was also assumed that the intracellular space was insulated from the blood, that is

$$
\frac{\partial \phi_{i}}{\partial n}=0
$$

along the endocardium. The initial condition was that the extracellular potential in the tissue and the potential in the blood mass and the probe were set to zero. The intracellular potential was set to $-85 \mathrm{mV}$, giving rise to a transmembrane potential in the tissue of $-85 \mathrm{mV}$. Lastly, the reference potential at the origin was set to zero.

The governing equations were solved using the finite volume method [8] and a forward Euler time stepping scheme [9]. Simulations were performed over a period of $700 \mathrm{~ms}$ with a time step of $0.02 \mathrm{~ms}$ for the solution of the ionic current model and $0.1 \mathrm{~ms}$ for the solution of the bidomain equations.

The following simulations were performed to compare catheter-based activation measurement with the true activation on cellular level. First a reference beat was simulated with no catheter included in the model. For this simulation, the reference activation time at each site at which 
the probe was placed was identified as the time at which the upstroke of the local transmembrane potential crossed the $-30 \mathrm{mV}$ level.

Next, twelve beats were simulated by rotating the catheter location in increments of $30^{\circ}$ azimuthal angle as described above. For each catheter location a bipolar electrogram was computed by taking the potential at electrode 1 (in contact with the tissue) minus potential at electrode 2 (adjacent to 1). Each of the twelve bipolar signals was filtered by applying a digital 10 to $200 \mathrm{~Hz}$ direct form II transposed Butterworth bandpass filter of order 2+2 [10].

At each catheter location, activation was measured by annotating the filtered bipolar signals. Different annotation modes were investigated. In this study we follow closely the terminology used in [2] for defining three different annotations at each catheter location:

- peak amplitude: the annotation of the absolute minimum in the filtered bipolar potential.

- down-stroke: the annotation of the steepest negative slope in the potential.

- first peak: the annotation of the local potential maximum in the time interval between stimulation and down-stroke.

\section{Results}

Figure 3 shows two examples of bipolar electrograms at azimuthal angles of $60^{\circ}$ and $120^{\circ}$, respectively. For the purposes of illustration, both sites are shown with the three annotations, as described above. Figure 4 shows activation time along the circular line defined by the twelve catheter locations. Activation time is depicted for all three annotations and for the reference. Early activation occurs near to the pacing point (azimuth zero). The activation wave front spreads in both directions (positive and negative azimuth) yielding latest activation in locations approximately opposite to the stimulation point. A time offset is observed for all three annotations. First peak annotations consistently yield the earliest estimates of activation time, while peak amplitude annotations yield the latest estimates of activation time.

As a consequence of these observations, in clinical application only one annotation must be used for a procedure because of the necessity of distinguishing between early and late activation. Note that in such an application a constant offset does not add any bias to the analysis. Therefore, the measures for qualitative comparison listed in Table 1 are defined such that offset does not enter the result.

From Table 1 it can be seen that the correlation between the different bipolar annotations and the reference onset of activation is highest for the first peak annotation. For the down-stroke and peak amplitude annotations the correlation is below 0.8 . However, for all three correlations a p-value of less than 0.01 was obtained.

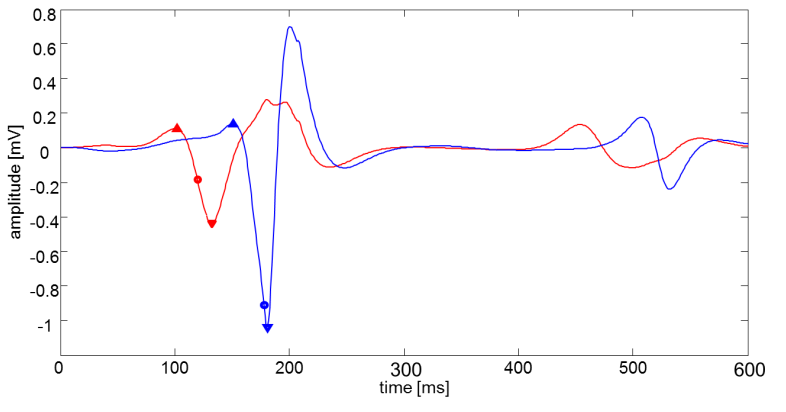

Figure 3. Filtered bipolar electrogram at $60^{\circ}$ (red) and at $120^{\circ}$ (blue) catheter rotation angle. Both signals display large early deflections which mark local activation and smaller late deflection corresponding to repolarisation. For both traces annotations (first peak $\triangle$, down-stroke $\circ$ and peak amplitude $\nabla$ ) are shown. Note that for all annotations activation is earlier at $60^{\circ}$ compared to $120^{\circ}$.

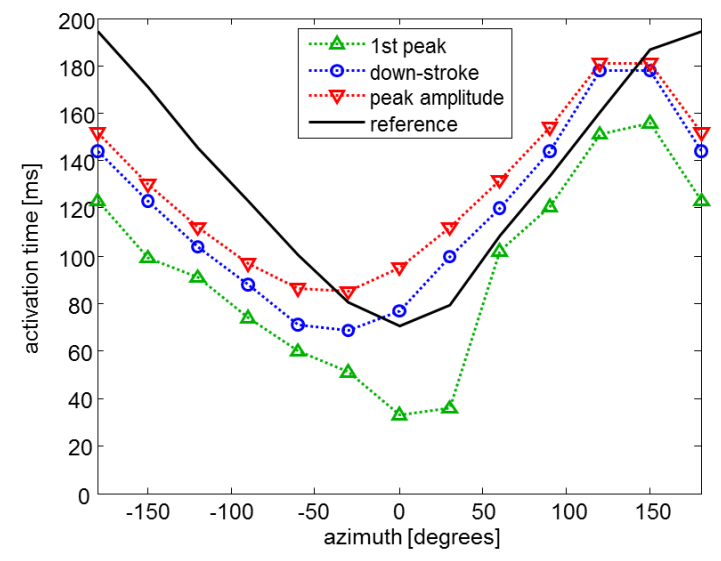

Figure 4. Activation time over azimuth for the three investigated annotations and the reference.

In Figure 4 a decreased correlation corresponds to a shift of the activation curves to the left. For two of the annotations, the onset of activation is erroneously observed at $-30^{\circ}$. Also root mean square (RMS) errors (Table 1) between normalised annotations and normalised reference activation were computed, where normalisation is defined such that the mean activation time of all twelve locations is set to zero (elimination of offset). Again, the first peak annotation performs best, however, the RMS-errors for all three annotations were above $20 \mathrm{~ms}$.

\section{Discussion}

Despite the fact that mapping of bipolar signals is a well-accepted gold standard for assessing cardiac activation, to the authors' knowledge the absolute accuracy of such methods has not been investigated thoroughly. This 
Table 1. Quantitative Comparison of Activation Measures

\begin{tabular}{|l|ccc|}
\hline & First Peak & Slope & Amplitude \\
\hline Correlation Coefficient & 0.866 & 0.790 & 0.762 \\
RMS-error (ms) & 21.0 & 25.5 & 26.6 \\
\hline
\end{tabular}

might be due to the fact that accurate reference activation data is not easily accessible in humans or in vivo. Computer modelling offers the possibility of accurately computing both bipolar signals and the underlying membrane activation for comparison purposes.

Our simulations confirm that catheter-based mapping displays significant features of cardiac activation. Areas of early and late activation are nicely distinguished. This is reflected by statistically significant correlations between 0.75 and 0.90 (Table 1). Furthermore, using models, the continuous spread of activation can be traced. However, the computed absolute accuracy is surprisingly poor, with RMS-errors of between 20 and $30 \mathrm{~ms}$, which amount to approximately $10 \%$ of the time required for activating the ventricle. Furthermore, for two of the annotations investigated, the position of the earliest activation (potential marker for an ablation target) is translated from the true position to the neighbouring catheter location.

Our simulations indicate that the first peak annotation, using an early portion of the signal related to ventricular activation, is the best mode for annotating catheter-based maps in humans. This is in agreement with recommended clinical practice [2], but in contradiction to theories relating down-stroke and negative amplitude to local activation [11]. This discrepancy might be explained by the fact that such theories do not consider the effect of filters needed for recording signals in a "noisy" clinical environment.

Furthermore, it is a general rule that geometrically large sensors have poor spatial resolution. Ablation catheters are difficult to design as they must perform a double function. Firstly, they deliver energy (requiring a large surface area) for controlled curative destruction of tissue, and, secondly, they measure signals (requiring smaller electrodes) for diagnostic purposes.

Our study quantifies the limited accuracy of current catheter-based activation mapping in an idealised model of the ventricle. Computed errors are surprisingly large considering that such mapping techniques are routinely used to guide catheter ablation of various types of arrhythmia. Results suggest that there is significant space for future improvement of activation mapping. This might be beneficial for improving treatment of complex types of arrhythmia.

\section{References}

[1] Cantwell CD, Roney CH, Ng FS, Siggers JH, Sherwin SJ, Peters NS. Techniques for automated local activation time annotation and conduction velocity estimation in cardiac mapping. Computers in Biology and Medicine 2015; 65:229-242.

[2] Munoz FDC, Buescher T, Asirvatham SJ. Teaching points with 3-dimensional mapping of cardiac arrhythmias taking points: Activation mapping. Circulation Arrhythmia and Electrophysiology 2011;4(3):e22-e25.

[3] Colli-Franzone P, Guerri L, Taccardi B. Modeling ventricular excitation: axial and orthotropic anisotropy effects on wavefronts and potentials. Mathematical Biosciences 2004; 188(1-2):191-205.

[4] Schmitt OH. Biological information processing using the concept of interpenetrating domains. In Leibovic KN (ed.), Information Processing in the Nervous System. New York: Springer-Verlag, 1969; 325-331.

[5] Ten Tusscher KHWJ, Bernus O, Hren R, Panfilov AV. Comparison of electrophysiological models for human ventricular cells and tissues. Progress in Biophysics and Molecular Biology 2006;90(1-3):326-345.

[6] Johnston BM. Six conductivity values to use in the bidomain model of cardiac tissue. IEEE Transactions on Biomedical Engineering 2016;63(7):1525-1531.

[7] Rush S, Abildskov JA, McFee R. Resistivity of body tissues at low frequencies. Circulation Research 1963;12:40-50.

[8] Johnston PR. A finite volume method solution for the bidomain equations and their application to modelling cardiac ischaemia. Computer Methods in Biomechanics and Biomedical Engineering 2010;13(2):157-170.

[9] Barnes JP. Mathematically Modelling the Electrophysiological Effects of Ischaemia in the Heart. Ph.D. thesis, Griffith University, December 2013.

[10] Bianchi G, Sorrentino R. Electronic Filter Simulation and Design. New York: McGraw-Hill, 2007.

[11] Steinhaus BM. Estimating cardiac transmembrane activation and recovery times from unipolar and bipolar extracellular electrograms: a simulation study. Circulation Research 1989;64(3):449-462.

Address for correspondence:

Peter Johnston, School of Natural Sciences and Queensland Micro- and Nanotechnology Centre, Griffith University

Nathan, Queensland, Australia, 4111, p.johnston@griffith.edu.au 